\title{
Development of Immunosensors
}

Based on Commercially Available Surface Acoustic Wave (SAW) Devices

\author{
N. Barié ${ }^{1}$, H. Sigrist ${ }^{2}$, M. Rapp ${ }^{1}$ \\ ${ }^{1}$ Forschungszentrum Karlsruhe GmbH, Institut für Instrumentelle Analytik, P.O. Box 36 40, D-76021 Karlsruhe, Germany \\ ${ }^{2}$ Centre Suisse d'Electronique et de Microtechnique, Jaquet-Droz 1, CH-2007 Neuchâtel, Switzerland
}

In the present paper we report about the development of immunosensors based on commercially available surface acoustic wave (SAW) devices. The SAW devices applied were originally developed for use in modern telecommunications and are thus available as series products at low costs.

For biosensing applications the devices must be coated with a shielding layer to prevent corrosion of the aluminum structures in aqueous media. Thin films of polyimide and parylene, respectively, show good shielding properties and were used as a basis for immobilization of receptor molecules.

Various approaches to coating the shielded surfaces with receptive biomolecules are presented briefly and discussed.

Finally, covalent binding of dextran as an intermediate layer on SAW devices is described. Dextran bonding is achieved by a photoimmobilization process. The aryldiazirine-functionalized bovine serum albumin (T-BSA) serves as a multifunctional light-activated linking agent (photolinker polymer). Dextran and the photolinker are mixed and photobonded to the sensor surface. Essential parameters of the coating procedure are optimized.

Immobilization of proteins to the dextran layer via carbodiimide chemistry is demonstrated. The feasibility of specific immunosensing is investigated using SAW sensors connected to a fluid handling system.

\section{Introduction}

Mass-sensitive electroacoustic devices have already proved suitable in many technical applications, above all in electronics, electric data processing and high-frequency technology. They are operated in the ultrasonic range at frequencies ranging from $10 \mathrm{MHz}$ to $2 \mathrm{GHz}$. For (bio)sensing, these devices are provided with a selective coating and installed into an oscillator circuit as a frequency-determining element. The interaction of the acoustic wave with the surrounding medium then causes the velocity and amplitude of the wave to change, which is indicated by a change in the resonance frequency and insertion loss of the oscillator. Thus, changes at the surface, such as changes in the mass, viscoelasticity, conductivity, dielectricity constant or temperature can be detected. However, the major measurement parameter generally is the change in the resonance frequency due to the change in mass, which results from the interaction between the analyte and the coating.

Sensors on the basis of various acoustic waves, which differ in terms of boundary conditions and the direction of their displacement components, have been implemented for both gaseous and liquid media, e.g. quartz crystal microbalances (QCM) [1-4] and sensors based on Rayleigh waves [5, 6], acoustic plate modes [7-9], surface transverse waves [10, 11] and Love waves [12-15].

The devices used for this work are commercially available "low-loss filters" which are generally applied as miniaturized high-frequency (HF) filter units or stabilizers in HF communications technology. Polymer-coated devices have already been applied in gas sensors for qualitative and quantitative analysis of organic solvent mixtures [16, 17]. Problems of food, cosmetics and tobacco industry are being examined with a view to establish a quality control during production, based on the distinction of different aromas [18]. Recently, the devices were tested for use in liquid phases, in particular for biosensing.

\section{Shielding Layers for Commercially Available SAW Devices}

In the past years, various strategies for the production of biosensors on the basis of acoustic mass-sensitive sensors were studied extensively and described in detail in literature [14, 19-21]. These descriptions, however, focused on devices of own construction with gold structures that were adapted to the specific measurement problem. In this work commercially available SAW devices shall be applied as mass-sensitive biosensors. These devices are produced in series at low costs for telecommunications purposes. Their structures and bonding wires are made of aluminum. 
For biosensing the surface of the device must be covered by a biosensitive layer of proteins, by means of which the analyte molecules can be identified in the sample. The reactions take place in aqueous media. Immobilization processes may even require acid, basic or chloride-containing surroundings. Due to these aggressive chemical conditions, the aluminum structures on the device have to be protected. Coating of the complete device with a polymer shielding layer has proved to be the most simple and suitable method [22]. This coating is to ensure a sufficient protection of the aluminum against corrosive attacks by the surrounding media without modifying the acoustic properties.

In this work, poly-(2-chloro-p-xylylene) ("parylene C") and a polyimide consisting of the monomers p-phenylenediamine and 3,3',4,4'-biphenyltetracarboxylic dianhydride were applied as polymer coatings. In principle, both polymers, polyimide and parylene, are suited for use as shielding layers in biosensing with SAW devices. Critical study, however, shows that the parylene coating has a number of advantages compared to the spin coated polyimide film [23].

- Due to the coating process applied, reproducibility of parylene coating is far better than that of polyimide spin coating.

- For the same reason, parylene coating leads to very smooth films, which is a major requirement for use on SAW devices.

- This method also ensures protection of the bonding wires which are enclosed by parylene on all sides, whereas only insufficient enclosure is achieved by polyimide.

- The parylene coating ensures excellent protection against corrosion. Consequently, the SAW devices can be operated in aqueous media and under aggressive conditions for a longer period of time than devices with polyimidecoated surfaces.

Due to these advantages of parylene coating, the field of application of the thus protected SAW devices is wider than that of polyimide-coated devices.

\section{Immobilization of Biomolecules on SAW Sensor Surfaces}

Various possibilities exist for the modification of SAW devices to biosensors [24, 25]. In general, biomolecules are immobilized on the sensor surface and then undergo reactions with the corresponding analytes.

A survey of the approaches to biofunctionalization of the shielded surface of commercially available SAW devices is given below.

\section{Physical adsorption of proteins on the sensor surface}

One of these approaches consists in the physical adsorption of biomolecules on the surface. These biomolecules then react with the respective receptive molecules [26]. However, this method has the drawback of an adsorption on the surface being associated with small binding energies. This means that the adsorbed biomolecules can be desorbed easily, as a result of which it is rather difficult to obtain a stable receptor layer.

It was therefore searched for alternatives to achieve covalent bonding of the proteins on the sensor surface. In biochemistry a variety of immobilization methods exist, but most of them work under aggressive conditions and, hence, cannot be applied to the commercially available SAW devices with aluminum structures.

\section{Cyano transfer technique}

The cyano transfer technique represents a modified version of the bromocyano immobilization known in biochemistry. Here the shielding layer is activated by means of cyanogen bromide. Upon activation the receptor molecules are bonded by carbodiimide [27].

This method, however, has the above drawback of working under rather drastic conditions. A relatively high number of sensors has to be rejected as the aluminum structures are attacked in spite of their shielding layer. In addition this method represents a rather long immobilization process which takes nearly one week from coating until the sensor is ready for use. Reproducibility is bad, which is attributed to the high number of working steps required for the application of the receptor layer and to the possible deactivation of the receptive molecules during carbodiimide activation. Last but not least, cyanogen bromide is a very toxic, highly reactive reagent that may only be handled with the respective protective measures being taken.

\section{Photoimmobilization of proteins}

Photoimmobilization of proteins on polyimide-coated SAW devices was demonstrated for two model substances (photoactive BSA or GOD derivatives) [28]. Co-immobilization of GOD using photoactive BSA did not meet with success.

Contrary to the cyano transfer technique, photoimmobilization has the advantages of covalent bonding taking place under soft conditions, no hazardous chemicals being used and taking a maximum of 2 hours only. A decisive drawback of this method results from the fact that the proteins to be immobilized first have to be provided with the photoactive group. This modification of the proteins is far from trivial as the production of the photoactive group alone requires a synthesis of several steps [29].

The two latter immobilization methods are relatively time- and labor-consuming and hardly transferable to other applications as they require special modifications of the sensor surface or the proteins to be immobilized. Further studies were therefore aimed at finding a universal method for surface biofunctionalization based on a standardized procedure that allows a rapid and reproducible immobilization of various biomolecules without special methods being required for the respective protein. Such a method can be achieved by covalent bonding of a dextran layer on the sensor surface [30]. 


\section{Immobilization of proteins on dextran-coated surfaces}

Various biosensor systems which are mostly based on optical signal transduction are presently available and fulfil the above requirements [31]. These systems are operated among others with dextran-coated sensor surfaces which may be modified easily and rapidly with proteins using a simple standard procedure. Bonding of the dextran layer generally takes place via 1, $\omega$-hydroxythiols which form self-assembled monolayers on the gilded sensor surface [32-34].

It seems to be reasonable to also apply such a dextran coating to mass-sensitive SAW devices in order to ensure a highly flexible use of these devices as biosensors. Here, however, gold structures are lacking and coating of the device with a thin gold film has not proved advantageous. Therefore, photoimmobilization [35] has been applied for the immobilization of the dextran layer on the coated SAW surface. This promising new method will be discussed more detailed in the following.

\section{Immobilization of Dextran to the Surface}

For covalent binding of dextran to the coated sensor surface, a photoactive polymer acts as a photolinker between dextran and the shielding layer [36]. In the present example, bovine serum of albumin, multiply derivatized with light-sensitive trifluoromethyl-aryldiazirine (T-BSA) [37], serves as multifunctional photolinker. T-BSA and dextran are applied to the coated SAW device. Under irradiation with light of the wavelength of $348 \mathrm{~mm}$, nitrogen is eliminated by the diazirine ring of T-BSA and a triplet carbene is generated. It inserts into a number of chemical compounds, preferably into $\mathrm{C}-\mathrm{H}, \mathrm{C}-\mathrm{C}, \mathrm{C}=\mathrm{C}, \mathrm{N}-\mathrm{H}, \mathrm{O}-\mathrm{H}$ and $\mathrm{S}-\mathrm{H}$ bonds [29]. As

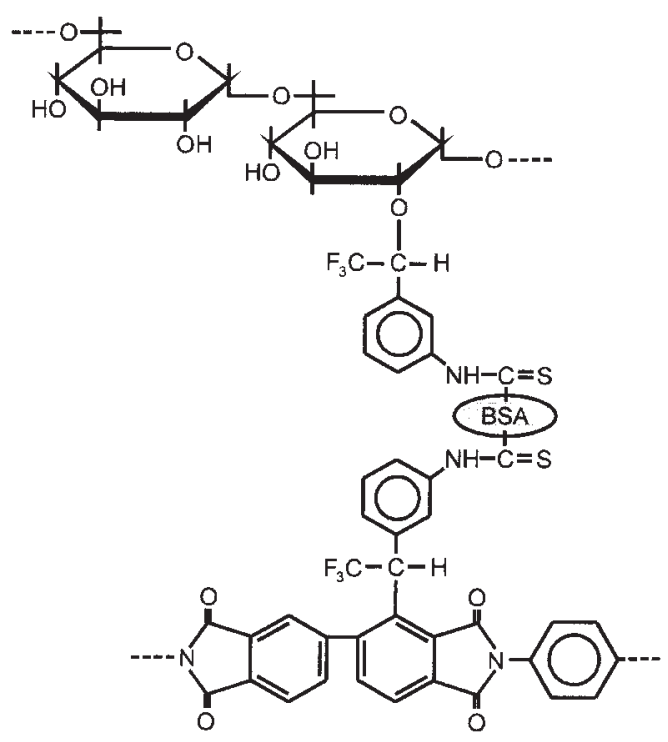

Dextran

T-BSA

Figure 1. Scheme of photolinker-polymer-mediated covalent immobilization of dextran to polyimide. The photobonding procedure is not restricted to polyimide.

Polyimide there are up to 10 TRIMID units per BSA molecule [38], the triplet carbenes may cause bonding with both the shielding layer and dextran by simultaneous insertion. Thus, covalent binding of dextran to the surface is achieved (see figure 1).

This immobilization method has the advantage of being a very simple and rapid procedure. The presence of functional groups on the sensor surface or the dextran is not required. Immobilization is possible on nearly any surface material [37]. Additionally T-BSA also serves to block nonspecific binding sites on the coated surface.

\section{Immobilization of Biomolecules on the Dextran-coated Surface}

In our work, we used biotinylated dextran and dextran initially modified with carboxymethyl groups by way of example.

Biotinylated dextran served to optimize the photoimmobilization procedure by quantifying bound $\left[{ }^{35} \mathrm{~S}\right]$-streptavidin with liquid scintillation counting.

Various biomolecules (hereinafter called ligands) can be bound covalently to sensor surfaces coated with carboxymethylated dextrans. The negatively charged dextran surface in the corresponding $\mathrm{pH}$ range serves the purpose of ligand enrichment in the three-dimensional hydrogel matrix. Covalent bonding may take place by a number of immobilization methods [30, 39-44]. The selection of a suited method is determined among others by the character of the ligands and the purpose of immobilization.

The most frequently applied immobilization method on carboxymethylated dextran is amino coupling with the activation of the carboxyl groups taking place by $\mathrm{N}$-hydroxysuccinimide (NHS) and N-(3-dimethylaminopropyl)-N-ethylcarbodiimide (EDC) via carbodiimide chemistry. The thus formed NHS esters react with nucleophilic groups of the ligand under elimination of NHS and formation of a covalent bond $[30,39]$. This method has the advantage of taking place under relatively soft conditions and allowing an easy and rapid immobilization. The nucleophilic groups considered are mainly primary amino groups, such as those existing at the $\mathrm{N}$-terminal and in lysine residues when using proteins and peptides. But also the thiol group of cysteine and to a certain degree the imidazole group of histidine and the hydroxyphenyl group of tyrosine may be applied for immobilization.

\section{Experimental}

\section{Materials and Instrumentation}

Dextrans were obtained from Molecular Probes, Oregon, USA (D-1856: dextran, biotin, 10,000 MW; D-1953: dextran, carboxymethyl, 70,000 MW). Urease was obtained from Biotrend Chemikalien $\mathrm{GmbH}$, Köln, Germany. Monoclonal anti urease antibodies (Clone No. UR-25), 
L-tryptophan and glucose oxidase were purchased from Sigma Chemical Company. N-hydroxysuccinimide (NHS), $\mathrm{N}$-(3-dimethylaminopropyl)-N-ethylcarbodiimide (EDC), Tween 20, KSCN and ethanolamine were obtained from Merck KGaA, Darmstadt, Germany. BSA (11924) was purchased from Serva Feinbiochemica GmbH, Heidelberg, Germany. $\left[{ }^{35} \mathrm{~S}\right]$-streptavidin was obtained from Amersham, Zürich, Switzerland. Polyimide (PI 2610) and promoter (VM 651) were kindly released by DuPont, Germany. Parylene coating was done by Comelec SA, La Chaux-de-Fonds, Switzerland. T-BSA was synthesized as described in [37]. All other reagents were of analytical grade.

The phosphate buffers applied $(0.5,20$ and $50 \mathrm{mM})$ were produced from $\mathrm{NaH}_{2} \mathrm{PO}_{4} * \mathrm{H}_{2} \mathrm{O}$ in the respective molarity. The $\mathrm{pH}$ value was set to $\mathrm{pH} 7.2$ with $\mathrm{KOH}$. $50 \mathrm{mM}$ phosphate buffer was used to produce $50 \mathrm{mM} P B S$ by addition of $150 \mathrm{mM} \mathrm{NaCl}$. The $P B S /$ Tween required for washing consisted of $50 \mathrm{mM}$ PBS with 0.02 vol.\% Tween-20.

A liquid scintillation counter (Tri-Carb Model 2300TR, Packard Instrument Company) was used for quantification of $\left[{ }^{35} \mathrm{~S}\right]$-labeled streptavidin in $5 \mathrm{ml}$ scintillation fluid (Ultima Gold, Packard No. 6013329).

For irradiation a Stratalinker 2400 UV Crosslinker (Stratagene $\mathrm{GmbH}$, Heidelberg, Germany) equipped with five $365 \mathrm{~nm}$ (major emission) UV light bulbs (F $158 \mathrm{BL}$; $15 \mathrm{~W}$ black light each) was employed. The coated devices were placed $4 \mathrm{~cm}$ apart from the light source and illuminated with an irradiance of $0.7 \mathrm{~mW} \mathrm{~cm}-2$.

The used SAW devices are commercially available lowloss filters based on horizontal polarized shear waves (HPSW, STW) on a $36^{\circ}$ rotated $\mathrm{LiTaO}_{3} \mathrm{XY}$-cut substrate. They were supplied by MuRata Company, Japan, type SAF380T with an operating frequency of $380 \mathrm{MHz}$. The typical attenuation during operation is only $2 \mathrm{~dB}$ in air and $4 \mathrm{~dB}$ in water [26]. The devices were placed in a TO8 chamber and built in a teflon adapter, via which the sensor was connected to the fluidic system. The liquid was pumped through the fluidic system by a peristaltic pump (Ismatec sa). A 4-port valve (Latek-TMV) was used to switch between the two solutions, i.e. buffer and analyte.

Measurements were done in an oscillator circuit. Resonance frequencies were measured by a Hewlett Packard frequency counter (HP-PM 6680), scanner (HP-PM 2327) and switch (HP-PM 2301). These instruments were controlled by a personal computer via a GPIB interface.

\section{Methods}

For optimization of the coating procedure, parylene-coated glass platelets $(5 \mathrm{~mm} \times 5 \mathrm{~mm})$ were applied. Polyimidecoated and parylene-coated SAW devices were used for sensor measurements.

\section{Surface passivation}

Prior to coating with polyimide or polystyrene, the surfaces were washed by immersion into $50 \mu$ l acetone (incubation time: $15 \mathrm{~s})$ and subsequent spinning off the solvent (2000 rpm; $10 \mathrm{~s})$. Then drying took place for $10 \mathrm{~min}$ at 30 mbar (RT).

Coating with polyimide was accomplished by a spin coating process [22]. $5 \mu 1$ aminopropyl-triethoxysilane were mixed with $9.5 \mathrm{ml}$ methanol and $500 \mu \mathrm{l}$ bidistilled water and stirred for 15 minutes. The thus prepared solution was allowed to stand for $12 \mathrm{~h}$ at room temperature. $50 \mu \mathrm{l}$ of the silane solution were applied to the surface and spinned off after an incubation time of $30 \mathrm{~s}(3000 \mathrm{rpm} ; 30 \mathrm{~s})$. The polyadduct of the monomers of p-phenylene-diamine and 3,3',4,4'-biphenyltetracarboxylic dianhydride was dissolved in N-methylpyrrolidone at a concentration of $650 \mu \mathrm{g} / \mathrm{ml}$. $50 \mu \mathrm{l}$ of the polymer mixture were pipetted onto the sensor and spinned off after an incubation time of $70 \mathrm{~s}$ (5000 rpm; 30 s). Subsequently, the coated device was subjected to annealing for $30 \mathrm{~min}$ at $200^{\circ} \mathrm{C}$ and for another $30 \mathrm{~min}$ at $350^{\circ} \mathrm{C}$. Then the coated sensor was cooled down slowly to room temperature.

Coating with poly-(2-chloro-p-xylylene) ("parylene C") was carried out in a vacuum coating facility by Comelec sa, Switzerland. Coating thickness was $0.8 \mu \mathrm{m}$.

\section{Immobilization of dextran}

T-BSA and dextran were dissolved in $0.5 \mathrm{mM}$ PBS (pH 7.2). A mixture of the T-BSA and dextran solution was applied to the coated surface (glass platelets: $20 \mu \mathrm{l}$; SAW devices: $6 \mu \mathrm{l})$. Following drying at reduced pressure $(2 \mathrm{~h}$, room temperature) the coated carriers were exposed to light for $45 \mathrm{~min}$. Then washing took place using chaotrop / detergentcontaining solutions (3 M KSCN, PBS/Tween). Incubation in the respective washing solution took place for $5 \mathrm{~min}$ each at room temperature.

For control purposes other glass platelets were subjected to an identical treatment, but not to an exposure.

\section{Optimization of the coating procedure}

For optimization of the coating procedure, parylene-coated glass substrates were modified with biotinylated dextran as described above. An aqueous solution of $\left[{ }^{35} \mathrm{~S}\right]$-streptavidin $(20 \mu \mathrm{l})$ was applied to the coated surface and incubated at room temperature for $1 \mathrm{~h}$. After washing with PBS/Tween (5 times) and water (3 times), bound $\left.{ }^{35} \mathrm{~S}\right]$-streptavidin was quantified by liquid scintillation counting in $5 \mathrm{ml}$ scintillation fluid each.

Each measurement comprised a double determination of the radioactivity of the streptavidin solution applied as well as a double determination of the predosage level.

\section{Immobilization of proteins on dextran}

Aqueous solutions of NHS (100 mM) and EDC (400 mM) were mixed, $5 \mu \mathrm{l}$ reagent mixture was applied to the dextran-coated sensor surface and incubated for $10 \mathrm{~min}$. After this activation of the dextran carboxyl functions, $10 \mu \mathrm{l}$ of protein solution in $10 \mathrm{mM}$ acetate buffer $(\mathrm{pH}$ 5.0) was 
applied and incubated at room temperature for $20 \mathrm{~min}$. Excess NHS esters were deactivated using $10 \mu \mathrm{l} 1 \mathrm{M}$ ethanolamine $(\mathrm{pH} 8.5)$. Unbound protein was washed off by repeated rinsing of the sensor surface with $50 \mathrm{mM}$ PBS ( $\mathrm{pH}$ 7.2). Non-specific binding sites were saturated with bovine serum albumin ( $4 \mathrm{wt} . / \mathrm{vol} . \%$ in $20 \mathrm{mM}$ PBS; $10 \mathrm{~min}$; room temperature). After a final washing step with $50 \mathrm{mM}$ PBS, the sensor was ready for biosensing.

\section{Monitoring of the immunoreaction}

During the experiments all buffer, reagent and protein solutions employed were kept at room temperature. The measurements with the SAW devices were performed under continuous fluid flow through the sensor (100 to $500 \mu \mathrm{l} / \mathrm{min}$ ). Time-dependent resonance frequency changes were monitored under constant flow conditions.

The coated SAW devices were installed in the flow system and rinsed with $20 \mathrm{mM}$ PBS until a constant resonance frequency $( \pm 100 \mathrm{~Hz})$ was reached. Solutions containing the antigen or non-specifically interacting (immuno-)reagents dissolved in PBS were injected into the carrier buffer. Following sampling rinsing with PBS took place.

\section{Results and Discussion}

\section{Photoimmobilization of Dextran}

Covalent bonding of dextran to the polymer-coated surfaces was achieved by co-immobilization with T-BSA as photolinker and surface coating with a mixture of T-BSA and dextran.

The coating procedure was optimized for bonding of a maximum amount of receptive protein to dextran. This is influenced directly by the amount of immobilized dextran. On the other hand, steric accessibility of the protein binding sites on the functionalized dextran plays a crucial role. To take into account the steric factor, the photoimmobilization parameters were optimized not by direct quantification of radioactively labeled dextran, but by the use of biotinylated dextran and sampling with $\left[{ }^{35} \mathrm{~S}\right]$-streptavidin following photoimmobilization. Then the amount of $\left[{ }^{35} \mathrm{~S}\right]$ bound by biotine-streptavidin bonds was measured using a scintillation counter. This assay mimics the general process of protein bonding to hydrogels, but in comparison to carbodiimidebased bonding procedures it has the advantage of being more rapid and excluding sources of error due to the following modification steps.

As a possible source of error, the multivalency of streptavidin, which may cause cross-linking of the biotinylated dextran chains, has to be taken into consideration. Since dextran is known to form swollen 3-dimensional hydrogels on surfaces, it is possible to also bind proteins to interior binding sites of this matrix. Due to cross-linking, diffusion processes in dextran may be inhibited and, consequently, the hydrogel capacity can no longer be used to the complete extent. The results (good reproducibility, small standard deviations) demonstrate, however, that this method is suited for optimization purposes.

For optimization it is only necessary to evaluate the results obtained within a single measurement series relative to each other and, thus, to determine the optimum value for the parameter investigated. For this reason, the dpm (decomposition per minute) values obtained in the radioactive measurements were not converted into absolute values of surface coverage. The higher the measured signal (dpm), the more streptavidin was bound and the better was the coating procedure.

\section{Optimization of the T-BSA/dextran ratio}

In the co-immobilization of binary systems the molar or mass ratio of both molecules is of decisive importance. To determine the optimum ratio in the co-immobilization of dextran with T-BSA, parylene-coated glass platelets were covered with various mixtures of biotinylated dextran (MW $10,000)$ and T-BSA. Then sampling was performed with $\left[{ }^{35} \mathrm{~S}\right]$-streptavidin.

As obvious from figure 2, the signal increases slowly with an increasing T-BSA fraction (and, hence, decreasing dextran fraction) as first a certain amount of the photolinker is supposed to exist for covalent bonding of the biotinylated dextran (and, hence, of $\left[{ }^{35} \mathrm{~S}\right]$-streptavidin). In case of very high proportions of T-BSA in the mixture, the signal drops drastically since the dextran fraction is constantly reduced until no dextran exists in the solution at $100 \mathrm{~mol} \%$ T-BSA. In the range between 71 and 86 mol\% T-BSA (i.e. 29 and

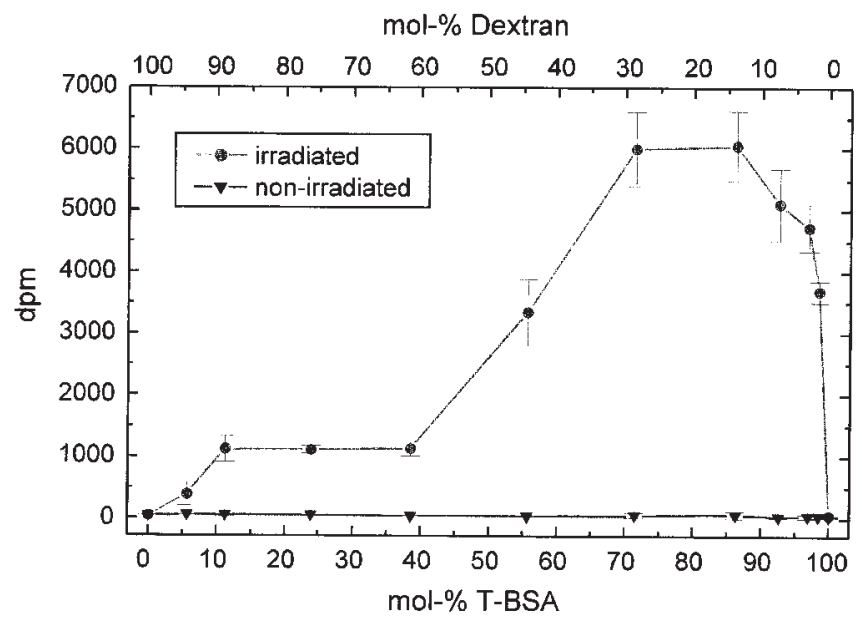

Figure 2. Optimization of the dextran to T-BSA ratio. Paryleneshielded glass platelets were coated with variably composed mixtures of biotinylated dextran and T-BSA, dried and exposed to light for $\mathbf{4 5} \mathrm{min}$. Control samples were not irradiated. Bonding was determined by measuring the radioactivity after the reaction with $\left[{ }^{35} \mathrm{~S}\right]$-streptavidin by liquid scintillation counting. (Mean values of three measurements). 


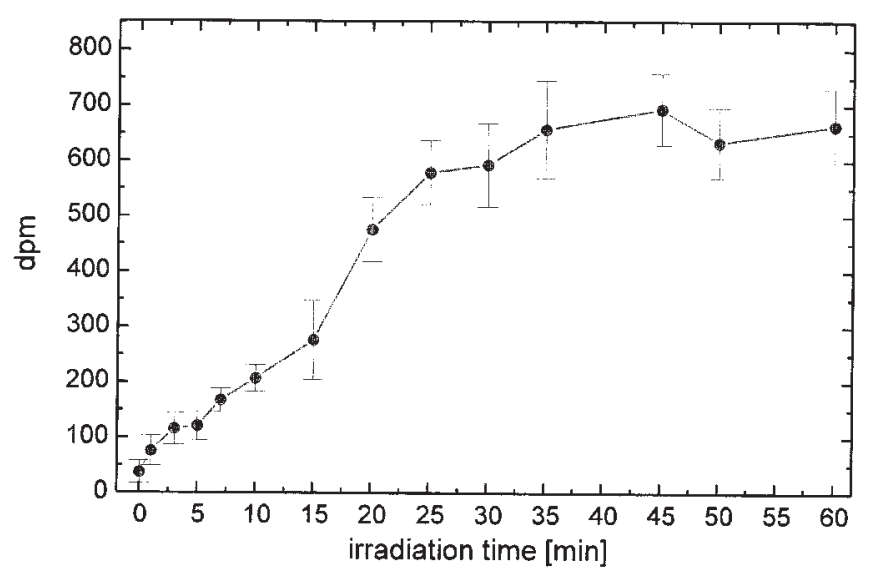

Figure 3. Optimization of irradiation time. Parylene-coated glass platelets were coated with a mixture of $25 \mathrm{~mol} \%$ biotinylated dextran and $75 \mathrm{~mol} \%$ T-BSA and exposed to light for the indicated period of time. (Mean values of three measurements).

14 mol\% dextran) a maximum occurs. This means that here the maximum amount of dextran is immobilized.

It is demonstrated by the unexposed control samples as well as by all following experiments that the test series is not affected by non-specific adsorption and that the process is clearly light-induced.

\section{Optimization of irradiation time}

As shown in figure 2 by the comparison of irradiated and not irradiated samples, co-immobilization of dextran with TBSA is a light-induced process. Irradiation time was optimized by applying a mixture of $25 \mathrm{~mol} \%$ biotinylated dextran and $75 \mathrm{~mol} \%$ T-BSA to the parylene-coated glass platelets. Following drying under vacuum the platelets were irradiated for variable periods of time. The data obtained by scintillation counting upon washing and sampling with $\left[{ }^{35} \mathrm{~S}\right]-$ streptavidin are represented in figure 3 . The signal first increases with increasing irradiation time as the amount of immobilized biotinylated dextran and, hence, of $\left[{ }^{35} \mathrm{~S}\right]$-streptavidin increases. No additional bonding of dextran is achieved after an irradiation time of $45 \mathrm{~min}$.

\section{Long-term stability during storage}

The dextran-coated surfaces are to serve as a basis for the immobilization of biomolecules. It is therefore reasonable to provide a large number of devices with, if possible, identical dextran coatings and to store the thus coated devices until use.

To study the long-term stability of the dextran coating during storage, parylene-coated glass platelets were coated, exposed, washed and dried and finally stored in the dried state for up to 190 days at $5^{\circ} \mathrm{C}$ in the refrigerator. Prior to scintillation measurement, the samples were rinsed with $20 \mathrm{mM}$ PBS for $30 \mathrm{~min}$ to enable swelling of the dextran

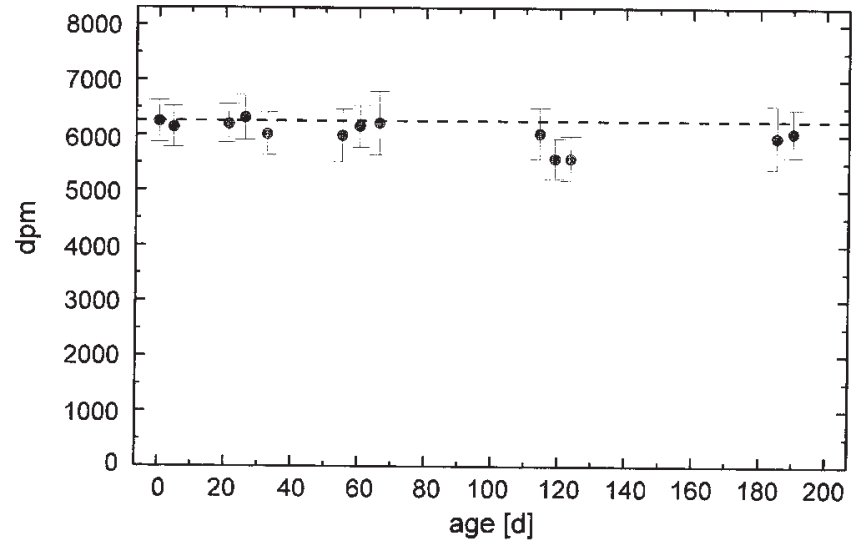

Figure 4. Investigation of long-term stability of the dextran coating during storage in the dried state at $5^{\circ} \mathrm{C}$. (Mean values of three measurements)

layer and then subjected to washing again (3 times each with $3 \mathrm{M} \mathrm{KSCN}$ and PBS/Tween).

In figure 4 the scintillation measurement data obtained are represented as a function of storage time. Over the entire test duration, no decrease in the signal and, hence, in the immobilized amount of dextran can be observed. This means that dry storage of dextran-coated devices under the given conditions is possible for at least half a year.

\section{Immobilization of Biomolecules on the Dextran-coated Surface}

For the immobilization of biomolecules on dextran-coated surfaces a number of methods exist. They have been investigated and described in various publications [30, 39-45]. One of these methods was taken and adapted to SAW sensors.

The immobilization of biomolecules by carbodiimide chemistry (amide coupling) [39, 40, 42, 43, 45] is reflected by the sensor response, as shown in figure 5 by the example of an immobilization of monoclonal anti-urease antibodies. Here, the SAW sensor coated with carboxymethylated dextran was first rinsed with the buffer solution for recording the base line. Then 1-step activation with EDC and NHS took place. After this sampling was performed with the solution of anti-urease antibodies in the immobilization buffer. Subsequently, deactivation of excess NHS esters by ethanolamine was performed and the non-specific binding sites were blocked with BSA.

The change of frequency $\Delta \mathrm{f}$, which can be noticed during washing with the basic buffer after the deactivation step, is attributed to the covalent bonding of the ligand and, thus, serves to control the immobilization procedure. 


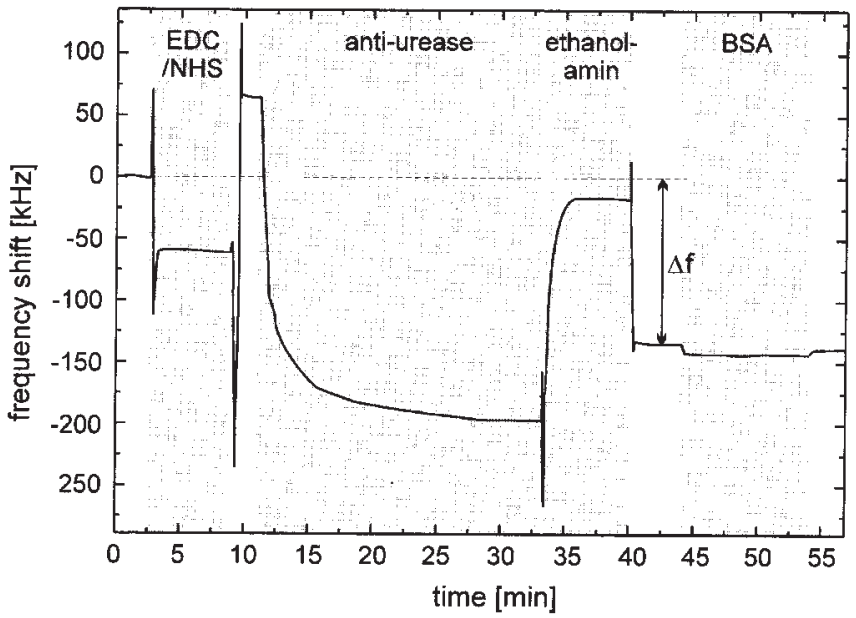

Figure 5. Sensor response during immobilization of ligands on carboxymethylated dextran (shielding layer: polyimide; operating frequency: $378.54 \mathrm{MHz}$; insertion loss: $5.64 \mathrm{~dB}$; buffer: $20 \mathrm{mM}$ PBS with $140 \mathrm{mM} \mathrm{NaCl}$, pH 7.2; $400 \mathrm{mM}$ EDC, $100 \mathrm{mM}$ NHS; $15 \mu \mathrm{g} / \mathrm{ml}$ monoclonal anti-urease antibodies in $10 \mathrm{mM}$ acetate buffer, $\mathrm{pH}$ 5.0; $1 \mathrm{M}$ ethanolamine; $1 \mathrm{mg} / \mathrm{ml}$ BSA).

\section{Immunological Studies}

\section{Immunoreaction and specificity}

As the proportion of non-specific binding of protein on dextran is negligibly small, the specificity of the immunosensor is mainly determined by the specificity of the receptive molecules applied. This influence is illustrated by the example of dextran-coated SAW devices which were modified with monoclonal anti-urease antibodies. Sampling of the sensors was performed with specific and non-specific antigens and the resulting frequency changes were observed. The corresponding sensor signals are represented in figure 6.

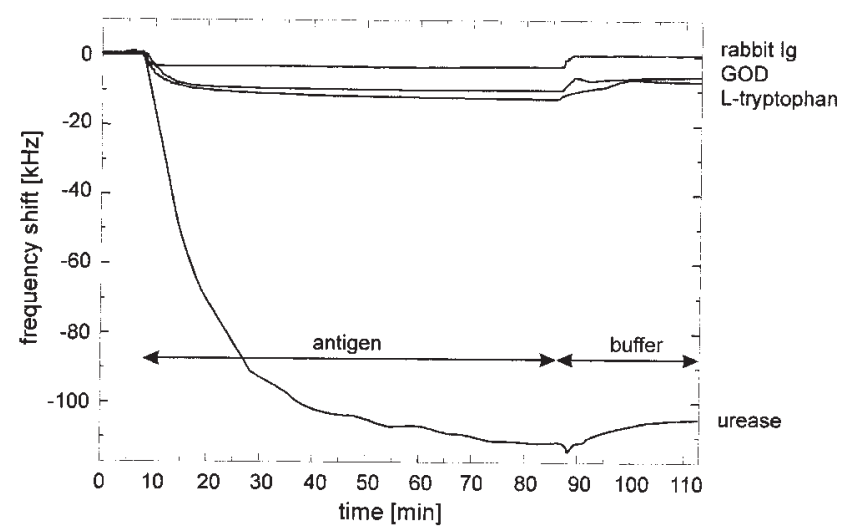

Figure 6. Specific and non-specific reactions on a SAW device coated with carboxymethylated dextran and modified with monoclonal anti-urease antibodies (20 mM PBS with $140 \mathrm{mM} \mathrm{NaCl}$, pH 7.2; $65 \mu \mathrm{g} / \mathrm{ml}$ urease, rabbit Ig, GOD, L-tryptophan each).

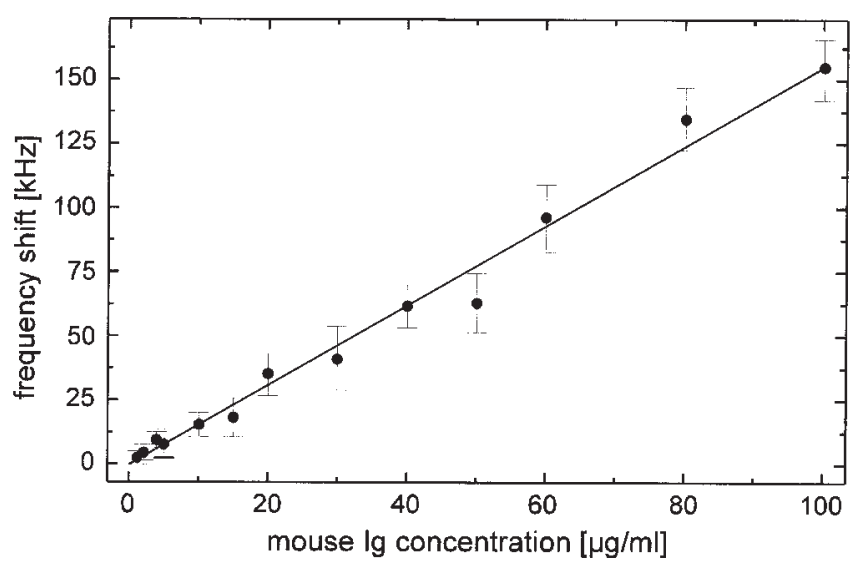

Figure 7. Sampling SAW devices coated with carboxymethylated dextran and modified with anti-mouse Ig antibodies with solutions of mouse $\mathrm{Ig}$ in various concentrations. (Mean values of three measurements).

The specific reaction with urease led to a frequency change of about $105 \mathrm{kHz}$. Sampling with non-specific antigens (L-tryptophan, GOD, rabbit Ig), however, only yielded small frequency changes of no more than $8 \%$ of the specific signal.

The ratio between the specific and the non-specific signal is a measure of the specificity of the respective immunosensor. In this case of L-tryptophan or GOD and urease, a ratio of about 14 is found, which confirms the high specificity of the immunosensor.

To investigate the reproducibility of the sensor signals in the immunoreaction, five dextran-coated SAW devices were coated with monoclonal anti-urease antibodies and sampling took place with solutions of $65 \mu \mathrm{g} / \mathrm{ml}$ urease in PBS. A mean frequency change of $110 \mathrm{kHz}$ with $12 \%$ scattering was achieved.

\section{Detection limit}

It is evident from figure 7 (system mouse-Ig / anti-mouse $\mathrm{Ig}$ ) that the change in resonance frequency increases linearly with the mouse-Ig concentration in the concentration range studied. The slope of the curve is $1.56 \mathrm{kHz} / \mu \mathrm{g} \mathrm{ml}^{-1}$ (correlation coefficient 0.991). Taking into account the frequency noise of about $40 \mathrm{~Hz}$ during operation with PBS, a minimum antigen concentration of $70 \mathrm{ng} / \mathrm{ml}$ can be detected with the present immunosensor. The detection limit of the sensor is $7 \mathrm{pg} / \mathrm{mm}^{2}$.

\section{Summary and Conclusion}

To sum up, it may be pointed out that after application of a polymer coating the commercially available and inexpensive 
SAW devices are suited for use as chemical or biochemical sensors. Various approaches to surface biofunctionalization have been presented.

To enable a highly flexible use of the SAW devices as biosensors, a dextran layer was immobilized on the sensor surface. The covalent photobonding of dextran to the surface was achieved by photolinker-mediated immobilization with aryldiazirine-functionalized bovine serum albumin. Process parameters were optimized for maximum protein binding. The optimal composition of the coating mixture was found to be about $25 \mathrm{~mol} \%$ dextran and $75 \mathrm{~mol} \% \mathrm{~T}$ BSA. The photoreaction was finished after 45 min irradiation.

The use of dextran as an intermediate layer for biosensor applications was shown by immobilization of monoclonal anti-urease antibodies on carboxymethylated dextran via carbodiimide chemistry and immunoreaction with urease. The thus generated dextran-coated biosensors were characterized among others by a high specificity and few non-specific adsorptions of biomolecules.

Thus, the engineering of a very versatile SAW biosensor has been attained. The method is applicable for simple, rapid and reproducible immobilization and detection of biomolecules.

\section{References}

1. Sauerbrey, G.Z. Z. Phys. 1959, 155; 206-222.

2. Shons, A.; Dorman, F.; Najarian, J. J. Biomed. Mater. Res. 1972, 6, 565-570.

3. Geddes, N.J.; Paschinger, E.M.; Furlong, D.N.; Ebara, Y.; Okahata, Y.; Than, K.A.; Edgar, J.A. Sens. Act. 1994, B17, 125-131.

4. Nakanishi, K.; Muguruma, H.; Karube, I. Anal. Chem. 1996, 68, 1695-1700.

5. Wohltjen, H., Dessy, R.E.; Anal. Chem. 1979, 51, 1458-1475.

6. D'Amico, A.; Palme, A.; Verona, E.; Sens. Act. 1982/83, 3, 3139.

7. Martin, S.J.; Ricco, A.J.; Niemczyk, T.M. ; Frye, G.C. Sens. Act. 1989, 20, 253-268.

8. Andle, J.C.; Vetelino, J.F.; Lade, M.W.; McAllister, D.J. Sens. Act. 1992, B8, 191-198.

9. Dejous, C.; Savart, M.; Rebiere, D.; Pistre, J. Sens. Act. 1995, B26-27, 452-456.

10. Thompson, M., Auld, B.A. Proc. IEEE Ultrasonics Symp. 1986, 261-266.

11. Baer, R.L.; Flory, C.A.; Tom-Moy, M.; Solomon, D.S. Proc. IEEE Ultrasonics Symp. 1992, 293-298.

12. Kovacs, G.; Vellekoop, M.J.; Haueis, R.; Lubking, G.W.; Venema, A. Sens. Act. 1994, A43, 38-43.

13. Du, J.; Harding, G.L.; Ogilvy, J.A.; Dencher, P.R.; Lake, M Sens. Act. 1996, A56, 211-219.

14. Harding, G.L.; Du, J.; Dencher, P.R.; Barnett, D.; Howe, E. Sens. Act. 1997, A61, 279-286.

15. Gizeli, E. Smart Mater. Struct. 1997, 6, 700-706.
16. Rapp, M.; Barié, N.; Stier, S.; Ache, H.J. Proc. IEEE Ultrasonics Symp. 1995, 477-480.

17. Barié, N.; Rapp, M.; Ache, H.J. Sens. Act. 1998, B46, 97-103.

18. Stier, S. Ph.D. Thesis, University of Karlsruhe, Germany, 1997.

19. Rickert, J.; Brecht, A.; Göpel, W. Anal. Chem. 1997, 69, 14411448.

20. Kondoh, J.; Matsui, Y.; Shiokawa, S.; Wlodarski, W. Sens. Act. 1994, B20, 199-203.

21. Tom-Moy, M.; Baer, R.L.; Solomon, D.S.; Doherty, T.P. Anal. Chem. 1995, 67, 1510-1516.

22. Wessa, T.; Barié, N.; Rapp, M.; Ache, H.J. Sens. Act. 1999, accepted.

23. Barié, N.; Wessa, T.; Rapp, M.; Sens. Act. 1999, submitted.

24. Turner, A.P.F.; Karube, I.; Wilson, G.S. in: Biosensors - fundamentals and application; Oxford University Press 1987, 8599.

25. Guilbault, G.G. Biotechnology 1989, 7, 349-351.

26. Rapp, M.; Moss, D.A.; Wessa, T.; Reichert, J.; Ache, H.J. Eurosensor VII; Budapest, Hungary, 1993.

27. Rapp, M.; Wessa, T.; Ache, H.J. IEEE Symposium 1995, Seattle.

28. Wessa, T.; Rapp, M.; Sigrist, H.; Ache, H.J. Conf. Proc. Biosensor'96 1996, Bangkok.

29. Dolder, M.; Michel, H.; Sigrist, H. J. Prot. Chem. 1990, 9, 407-415.

30. O'Shannessy, D.J.; Brigham-Burke, M.; Peck, K. Anal. Biochem. 1992, 205, 132-136.

31. Owen, V. Biosens. Bioelectr. 1997, 12, 1-2.

32. Troughton, E.B.; Bain, C.D.; Whitesides, G.M.; Nuzzo, R.G.; Allara, D.L.; Porter, M.D. Langmuir 1988, 4, 365-385.

33. Bain, C.D.; Troughton, E.B.; Tao, Y.-T.; Evall, J.; Whitesides, G.M.; Nuzzo, R.G. J. Am. Chem. Soc. 1989, 111, 321-335.

34. Löfas, S.; Johnsson, B. J. Chem. Soc. Chem. Commun. 1990, $21,1526-1528$.

35. Sigrist, H.; Collioud, A.; Clémence, J.F.; Gao, H.; Luginbühl, R.; Sänger, M.; Sundarababu, G. Optical Engineering 1995, 34, 2339-2348.

36. Barié, N.; Rapp, M.; Sigrist, H.; Ache, H.J. Biosens. Bioelectr. 1998, 13, 855-860.

37. Sigrist, H.; Gao, H.; Wegmüller, B. BioTechnology 1992, 10, 1026-1028.

38. Gao, H; Sänger, M.; Luginbühl, R.; Sigrist, H. Biosens. Bioelectr. 1995, 10, 317-328.

39. Johnsson, B.; Löfas, S.; Lindquist, G. Anal. Biochem. 1991, 198, 268-277.

40. Johnsson, B.; Löfas, S.; Lindquist, G.; Edström, A.; MüllerHillgren, R.M.; Hansson, A. J. Mol. Recogn. 1995, 8, 125-131.

41. O' Shannessy, D.J.; Wilchek, M. Anal. Biochem. 1990, 191, $1-8$.

42. Löfas, S.; Johnsson, B.; Tegendal, K.; Rönnberg, I. Coll. Surf. B: Biointerfaces 1993, 1, 83-89.

43. Karlsson, R.; Michaelsson, A.; Mattsson, L. J. Immunol. Meth. 1991, 145, 229-240.

44. Chaiken, I.; Rosé, S.; Karlsson, R. Anal. Biochem. 1992, 201, 197-210.

45. Yonezawa, S.; Kambegawa, A.; Tokudome, S. J. Immunol. Meth. 1993, 166, 55-61. 